Père M.-C., Dourmad J.-Y., Etienne M., 1995. Mise en évidence de la capacité utérine chez la truie. Journées Rech. Porcine en France, 27, 19-24.

Père M.-C., Dourmad J.-Y., Etienne M., 1996. Variation du débit sanguin utérin au cours de la gestation chez la truie. Journées Rech. Porcine en France, 28, 371-378.
Père M.-C., Dourmad J.-Y., Etienne M., 1997. Effect of number of pig embryos in the uterus on their survival and development and on maternal metabolism. J. Anim. Sci., 75, 1337-1342.

Reynolds L.P., Ford S.P., Ferrell C.L., 1985. Blood flow and steroid and nutrient uptake of the gravid uterus and fetus of sows. J. Anim. Sci., 61, 968-974.

\title{
Conséquences de l'augmentation de la prolificité des truies sur la survie et la croissance du porcelet
}

La prolificité des truies et le nombre de porcelets sevrés par truie et par an constituent le premier facteur de rentabilité d'un élevage de porcs. Les cochettes d'origine hyperprolifique sont en nombre croissant dans les élevages de production et permettent actuellement un gain de $10 \%$ par rapport aux contemporaines sur la taille de portée à la naissance. Ainsi, $18,5 \%$ des portées nées en 1995/1996 avaient une taille supérieure à 15 porcelets (J. Dagorn, communication personnelle). Mais ce progrès génétique considérable ne sera pleinement valorisé que lorsque l'on parviendra à mieux maîtriser la survie postnatale et la croissance des porcelets en surnombre. Après un bref rappel des principaux facteurs associés à une mortalité postnatale élevée, le texte présente les conséquences de l'augmentation de la taille de la portée sur la survie et la croissance des porcelets ainsi que les solutions envisageables pour mieux gérer les porcelets surnuméraires, avant de conclure sur les pistes de recherche à privilégier à l'avenir.

\section{1 / Mortalité néonatale}

Malgré l'amélioration des techniques d'élevage et l'approfondissement des connaissances en matière de nutrition et de physiologie de la truie et du porcelet, la mortalité néonatale est restée particulièrement élevée dans l'espèce porcine. Entre 1975 et 1994, elle a chuté de 18,7 à $17,9 \%$ des porcelets nés et de 15 à $12,7 \%$ des nés vivants. Ces chiffres masquent cependant une très importante hétérogénéité entre les élevages, les meilleurs éleveurs réussissant à réduire ces chiffres de 3 à 4 points. Dans plus des deux tiers des cas, cette mortalité se manifeste au cours des deux premiers jours de vie et est associée aux problèmes d'écrasement et de faiblesse des porcelets : animaux légers, de faible vitalité et/ou consommant une quantité insuffisante de colostrum (J. Dagorn, communication personnelle).

Les facteurs prédisposant le nouveau-né à une mortalité précoce ne seront que briève- ment rappelés, de nombreuses synthèses ayant été publiées sur le sujet (voir par exemple Herpin et Le Dividich 1995). La survie du porcelet dépend à la fois : 1 ) de son état physiologique à la naissance (poids et maturité) ; 2) de l'homogénéité des poids intra-portée, afin d'éviter une compétition excessive entre les animaux ; 3) des conditions de mise bas, une parturition prolongée s'accompagnant d'un risque accru d'asphyxie ou d'hypoxie transitoire ; 4) des conditions d'ambiance au sein de la case de mise bas, la sensibilité du nouveau-né au froid nécessitant son maintien à une température de $33-34{ }^{\circ} \mathrm{C}$ au cours du premier jour de vie ; 5) de son aptitude à ingérer rapidement une quantité suffisante de colostrum, source d'immunoglobulines et d'énergie ; et enfin 6) des qualités maternelles et des aptitudes laitières de la truie, afin de réduire les problèmes d'écrasement et d'optimiser la production de colostrum et de lait.

Qu'en est-il dans les lignées hyperprolifiques ? A notre connaissance, aucune étude scientifique ne s'est pour le moment spécifiquement attachée à préciser les conséquences de l'hyperprolificité sur la survie et la croissance des nouveau-nés. Nous tentons ci-dessous de décrire et d'analyser ces conséquences sur la base de quelques données expérimentales sur l'effet de la taille de la portée et des observations faites actuellement dans les élevages.

\section{2 / Conséquences de l'augmentation de la taille de la portée sur la survie et la croissance du porcelet}

L'augmentation de la taille de la portée s'accompagne d'une augmentation des pertes et d'une réduction de la croissance des porcelets, particulièrement marquées pour les portées de taille supérieure à 12 (Nielsen 1981 , Legault 1985). Ceci est confirmé par une
P. HERPIN, J. LE DIVIDICH

INRA Station de Recherches Porcines, 35590 St-Gilles 
enquête plus récente réalisée en Bretagne en 1992 (Pellois 1993). Ainsi, pour des portées de 8,12 et 15 porcelets la mortalité totale avant sevrage est respectivement de $12,5 \%, 16,5 \%$ et $24,7 \%$, et le gain moyen quotidien au cours des trois premières semaines de vie atteint $186 \mathrm{~g}, 166 \mathrm{~g}$ et $147 \mathrm{~g}$. La réduction de la croissance est probablement liée à la sévère compétition observée entre les porcelets et aux limites des capacités laitières de la truie, tandis que l'augmentation de la mortalité postnatale provient indirectement de la réduction du poids à la naissance, de la vitalité du nouveau-né et de l'augmentation de la durée de la mise bas.

\section{1 / Effet de la réduction du poids à la naissance}

Lorsque la taille de la portée augmente, la proportion d'animaux de poids inférieur à $1 \mathrm{~kg}$ augmente considérablement (Caugant et Guéblez 1993) et peut atteindre plus de $20 \%$ pour les portées de taille supérieure à 15 porcelets (England 1986). Cela s'accompagne aussi d'une augmentation de l'hétérogénéité des poids intra-portée, particulièrement défavorable pour la survie (English et Smith 1975). En suivant les performances de plus de 1800 porcelets issus de truies LWxLR et de verrats LWxP, Caugant et Guéblez (1993) ont clairement démontré ces effets en comparant les porcelets de poids inférieur à $1 \mathrm{~kg}$ aux porcelets dont le poids à la naissance est compris entre 1,5 et $2 \mathrm{~kg}$ : chez les porcelets légers, les pertes avant sevrage sont augmentées (36 contre $5 \%$ ), le poids au sevrage est plus faible de $27 \%$ (5,98 contre $8,15 \mathrm{~kg}$ ) et le GMQ global ainsi que le taux de muscle à l'abattage diminuent respectivement de $6,8 \%$ $(549$ contre $589 \mathrm{~g} / \mathrm{j})$ et 0,4 point $(55,2$ contre $55,6 \%)$.

L'effet défavorable d'un faible poids à la naissance s'explique à la fois par la réduction des capacités thermorégulatrices et par la plus faible vigueur des porcelets les plus légers. A cet égard, il convient de préciser que ces problèmes métaboliques ne se limitent pas au cas extrême des porcelets hypotrophes (moins de 800 g, Hayashi et al 1987) et peuvent aussi concerner des porcelets de poids relativement correct, compris entre 1 et $1,2 \mathrm{~kg}$ (P. Herpin, données non publiées). Ainsi, nous avons récemment montré que les capacités thermorégulatrices des porcelets LW à la naissance étaient altérées en-dessous d'un poids moyen d'environ $1,2 \mathrm{~kg}$ : le métabolisme de sommet, qui correspond à la production de chaleur maximale au froid, est réduit $(0,52$ contre $0,64 \mathrm{~kJ} / \mathrm{min} / \mathrm{kg}$ ), il est obtenu à une température ambiante plus élevée $(18,7$ contre $16 \square^{\circ} \mathrm{C}$ ) et le quotient respiratoire est plus élevé $(0,95$ contre 0,91$)$ indiquant une moindre utilisation des lipides. Ces porcelets sont donc plus fragiles à la naissance, une fragilité probablement encore accentuée par les phénomènes de compétition observés dans les portées de grande taille.

\section{2 / Effet de l'augmentation de la durée de mise bas}

La durée de la mise bas augmente avec la taille de la portée. Cela s'accompagne généralement d'une hausse de la mortinatalité, bien souvent en relation avec des problèmes d'asphyxie intra-utérine (Randall 1989). Ainsi, Dechamps et Nicks (1984) ont relevé 16,3\% de portées comportant des mort-nés pour des mise bas durant entre une et trois heures, contre $36,2 \%$ lorsque cette durée est comprise entre 3 et 5 heures. La pression partielle en $\mathrm{CO}_{2}$ du sang des porcelets nés vivants augmente aussi avec le nombre de porcelets nés par portée et l'ordre de naissance (Herpin et al 1996). Cela signifie que lorsque la mise bas se prolonge, ce qui peut être observé dans les portées nombreuses, le risque d'hypoxie intrautérine existe, même s'il n'est pas nécessairement mortel. Nos résultats suggèrent que dans ce cas, les porcelets ayant subit une hypoxie de parturition sont moins viables, qu'ils ingèrent du colostrum plus tardivement et que leur fonction de thermorégulation est altérée. Cela conduit à terme à une réduction du taux de survie (Herpin et al 1996).

Enfin, plus la mise bas est longue plus le statut immunitaire des porcelets est hétérogène. En effet, la teneur en immunoglobulines du colostrum décroît fortement au cours des heures qui suivent la mise bas : 6 heures après le début de celle-ci, les teneurs en IgG, en IgA et en IgM chutent respectivement de 32,26 et $24 \%$ (Klobasa et al 1987). Les derniers porcelets nés risquent donc d'acquérir une couverture immunitaire insuffisante et une moindre résistance aux maladies. De fait, les porcelets morts entre la naissance et le sevrage présentent généralement des teneurs sanguines plus faibles en immunoglobulines dès 14 heures de vie (Hendrix et al 1978).

\section{3 / Gestion des porcelets en surnombre}

Le constat de ce rapide tour d'horizon est simple : les performances de reproduction (survie et croissance) sont altérées lorsque la taille de la portée dépasse 12 à 13 porcelets. Les deux questions primordiales qui se posent actuellement sont donc les suivantes : comment assurer la survie d'un maximum de porcelets au cours des premières heures de vie, et comment élever les porcelets surnuméraires au cours des jours suivants?

\section{1 / Survie au cours des premières heures de vie}

Il ne s'agit pas ici de faire une liste exhaustive de toutes les solutions possibles, mais simplement de rappeler les éléments qui paraissent essentiels pour améliorer la survie, notamment dans le cas de l'hyperprolificité. Il va de soi tout d'abord que la conduite alimentaire des truies doit être raisonnée pour leur 
permettre d'atteindre la mise bas dans un bon état physiologique et nutritionnel. Le déclenchement, le contrôle de la durée et le suivi de la mise bas semblent indispensables pour éviter les problèmes évoqués plus haut et pour apporter soins et assistance aux porcelets à risque. Les mort-nés sont bien souvent des porcelets morts à la première observation de la portée et qui auraient certainement pu être sauvés à la naissance si une surveillance et des soins appropriés avaient pu être apportés. Enfin rappelons la nécessité d'assurer au porcelet un confort thermique optimal dès la naissance et un apport rapide et suffisant en colostrum.

\section{2 / Elevage des porcelets surnuméraires}

Une fois les portées homogénéisées (effectif, poids) au sein d'une bande de truies, les porcelets en excès (souvent choisis parmi les plus lourds) sont élevés par une truie nourrice de la bande précédente dont les porcelets ont été sevrés vers 21 jours de vie. Cette truie choisie pour ses qualités maternelles peut donc poursuivre une deuxième lactation. Toutefois, dans la majorité des cas, les porcelets sont ensuite sevrés à 6-8 jours d'âge. Les porcelets sevrés très précocement sont élevés dans un local chauffé à $30-32{ }^{\circ} \mathrm{C}$ et alimentés avec un lait de remplacement, parfois sous forme liquide (nourrice électronique), mais le plus souvent directement sous forme sèche. A notre connaissance, ces différentes méthodes n'ont fait l'objet d'aucune comparaison scientifique. Toutefois, selon Huysman et al (1994), les porcelets surnuméraires élevés par une truie adoptive jusqu'au sevrage auraient un taux de survie plus faible (92 contre $96 \%$ ) et une croissance plus forte (206 contre $122 \mathrm{~g} / \mathrm{j}$ ) que les porcelets sevrés entre 2 et 4 jours de vie. Dans tous les cas, le problème majeur est celui de l'acquisition d'une immunité satisfaisante par l'ingestion d'une quantité suffisante de colostrum.

\section{Conclusion}

Il est urgent de mettre en place des études spécifiques sur les conséquences de l'hyperprolificité sur la survie et l'élevage des porcelets. Des solutions spécifiques pour réduire la mortinatalité et accroître la viabilité et la vitalité des porcelets nés vivants sont actuellement à l'étude. En ce qui concerne l'élevage des porcelets surnuméraires, il est nécessaire d'acquérir des données sur un grand nombre d'animaux pour d'une part comparer les diverses méthodes d'élevage, et d'autre part déterminer la nature exacte des problèmes posés (nutritionnels, comportementaux, sanitaires). Au plan immunitaire, il faut déterminer la quantité minimale de colostrum assurant au nouveau-né une couverture suffisante en anticorps maternels (colostrum artificiel ?) et l'incidence d'une faible ingestion de colostrum sur la durée de la perméabilité de l'in- testin aux macromolécules. Dans le cas du porcelet sevré très précocement, on peut également s'interroger sur les conséquences de l'absence des IgA du lait maternel sur l'immunité locale et sur le développement de l'immunité active. Enfin, on n'a aucune information sur le devenir de ces animaux à plus long terme et notamment sur leur composition corporelle à l'abattage.

\section{Références}

Caugant A., Guéblez R., 1993. Influence du poids à la naissance du porcelet sur les performances ultérieures. Journées Rech. Porcine, 25, 123-128.

Dechamps P., Nicks B., 1984. Effects of non-infectious factors, particularly thermal comfort, on the viability and performance of piglets. Ann. Méd. Vét., $128,261-283$

England D.C., 1986. Improving sow efficiency by management to enhance opportunity for nutritional intake by neonatal piglets. J. Anim. Sci., 63, 1297 1306.

English P.R., Smith W.J., 1975. Some causes of death in neonatal piglets. Vet. Ann., 15, 95-104.

Hayashi M., Ingram D.L., Dauncey M.J., 1987. Heat production and respiratory enzymes in normal and runt newborn piglets. Biol. Neonate, 51, 324-331.

Hendrix W.F., Kelley F.W., Gaskins C.T., Hinrichs D.G., 1978. Porcine neonatal survival and serum gamma globulins. J. Anim. Sci., 47, 1281-1286.

Herpin P., Le Dividich J., 1995. Thermoregulation and the Environment. In : M.A. Varley (ed), The neonatal pig. Development and Survival, 57-95. CAB International, Wallington, UK.

Herpin P., Le Dividich J., Hulin J.C., Fillaut M., De Marco F., Bertin R., 1996. Effect of the level of asphyxia during delivery on viability at birth and early postnatal vitality of newborn pigs. J. Anim. Sci., 74, 2067-2075.

Huysman C.N., Roelofs P.F.M.M., Plagge J.G., Hoofs A.I.J., 1994. Motherless rearing or extension of the suckling period of piglets using " EMMA ". Proefverslag Proefstation voor de Varkenshouderig, $36,1-113$.

Klobasa F., Werhahn E., Butler J.E., 1987. Composition of sow milk during lactation. J. Anim. Sci., 64 1458-1466.

Legault C., 1985. La mortalité des porcelets de la naissance au sevrage : aspects génétiques. Porc Magazine, 174, 25-30.

Nielsen H.E., 1981. What are the real causes of piglet mortality. Guelph Porc Symposium, 14-15 Avril 1981, pp. 74-89, Guelph, Ontario.

Pellois H., 1993. Les performances techniques et technico-économiques en Bretagne : les progrès sensibles depuis 10 ans vont se poursuivre. A la pointe de l'élevage - Atout Porc, 266-28, 3-6.

Randall G.C.B., 1989. Form and development of the umbilical cord in pigs and their association with delivery of viable pigs. Am. J. Vet. Res., 50, 1512-1518. 